PROCEEDINGS OF THE

AMERICAN MATHEMATICAL SOCIETY

Volume 132, Number 8, Pages 2399-2409

S 0002-9939(04)07362-9

Article electronically published on March 24, 2004

\title{
HANKEL OPERATORS WITH ANTIHOLOMORPHIC SYMBOLS ON THE FOCK SPACE
}

\author{
GEORG SCHNEIDER
}

(Communicated by Joseph A. Ball)

\begin{abstract}
We consider Hankel operators of the form $H_{\bar{z}^{k}}: \mathcal{F}^{m}:=\{f$ : $f$ is entire and $\left.\int_{\mathbb{C} n}|f(z)|^{2} e^{-|z|^{m}}<\infty\right\} \rightarrow L^{2}\left(e^{-|z|^{m}}\right)$. Here $k, m, n \in \mathbb{N}$. We show that in the case of one complex dimension the Hankel operators are compact but not Hilbert-Schmidt if $m>2 k$.
\end{abstract}

\section{Preliminaries}

The investigation of Hankel operators on the Bergman space of certain domains $\Omega$ has a long history. See, for example, [24] and [25]. Furthermore, there have been some attempts to characterize compactness of Hankel operators on $L^{2}$ spaces of entire functions. In [26] the case of essentially bounded symbols is considered. This has the advantage that the corresponding Hankel operator is bounded.

There are interesting connections between the theory of partial differential equations and the theory of Hankel operators. In 12 it is shown that the canonical solution operator to $\bar{\partial}$ restricted to $(0,1)$-forms with coefficients in the spaces of holomorphic functions that are square integrable with respect to the weight function $e^{-|z|^{m}}, m \in \mathbb{N}$, can be interpreted as the Hankel operator with the symbol $\bar{\partial}$. For further connections to the $\bar{\partial}$-problem see [2], [1], [4], [16], [21], [27, [29], [12], 11] and [10].

We want to investigate operators of the form

$$
H_{f}: H_{f}(g)=(I-P)(f g) .
$$

Here $P$ denotes the Bergman projection

$$
P: L^{2}\left(\mathbb{C}^{n},|z|^{m}\right) \rightarrow \mathcal{F}^{m},
$$

with $L^{2}\left(\mathbb{C}^{n},|z|^{m}\right)=\left\{f: f\right.$ measurable and $\left.\int_{\mathbb{C}^{n}}|f(z)|^{2} e^{-|z|^{m}} d \lambda(z)<\infty\right\}$. Recall that the generalized Fock space $\mathcal{F}^{m}$ is the space of holomorphic functions that are square integrable with respect to the weight function $e^{-|z|^{m}}$. That is,

$$
\mathcal{F}^{m}=\left\{f: f \text { is entire and } \int_{\mathbb{C}^{n}}|f(z)|^{2} e^{-|z|^{m}} d \lambda(z)<\infty\right\} .
$$

Received by the editors October 25, 2002 and, in revised form, May 15, 2003. 2000 Mathematics Subject Classification. Primary 47B35; Secondary 32A15.

Key words and phrases. Fock space, Hankel operator, reproducing kernel. 
We use the following notation:

$$
\begin{aligned}
c_{k, m}^{2} & =\int_{\mathbb{C}^{n}}|z|^{2 k} e^{-|z|^{m}} d \lambda(z) \\
& :=\int_{\mathbb{C}^{n}}\left|z_{1}\right|^{2 k_{1}} \ldots\left|z_{n}\right|^{2 k_{n}} e^{-\left(\left|z_{1}\right|^{m}+\cdots+\left|z_{n}\right|^{m}\right)} d \lambda\left(z_{1}, \ldots, z_{n}\right) .
\end{aligned}
$$

Here $k=\left(k_{1}, \ldots, k_{n}\right)$ is a multi-index. We will abbreviate $c_{k, m}=c_{k}$ if no ambiguity arises.

It is well known $[3$ that

$$
\left\{u_{k, m}(z)=\frac{z^{k}}{c_{k, m}}\right\}
$$

constitutes a complete orthonormal system for $\mathcal{F}^{m}$. The reproducing kernel is given by

$$
K_{m}(z, w)=\sum_{k=1}^{\infty} \phi_{k}(z) \overline{\phi_{k}(w)},
$$

where $\left\{\phi_{k}\right\}_{k=1}^{\infty}$ is a complete orthonormal system of $\mathcal{F}^{m}$. It is known [3] that

$$
\begin{gathered}
K_{m}(z, \omega)=\bar{K}_{m}(\omega, z), \\
f(z)=\int_{\mathbb{C}^{n}} K_{m}(z, \omega) f(\omega) d \lambda(\omega) .
\end{gathered}
$$

Inserting our special orthonormal system into equation (1), we see that in the case $m=2$,

$$
K_{2}(z, w)=\sum \frac{z^{k}}{\sqrt{k !}} \frac{\bar{w}^{k}}{\sqrt{k !}}=e^{\bar{w} z} .
$$

We know 3 that the Bergman projection $P$ has the form

$$
P(g)(w)=\int_{\mathbb{C}^{n}} g(z) K_{m}(w, z) e^{-|z|^{m}} d \lambda(z) .
$$

Let us abbreviate $\frac{d \mu}{d \lambda}=e^{-|z|^{m}}$.

Until now the investigation of Hankel operators has mostly concentrated on essentially bounded symbols. That is,

$$
H_{f}: f \in L^{\infty}\left(\mathbb{C}^{n}\right) .
$$

For results see [24, [25] and [26].

Note that $L^{\infty}\left(\mathbb{C}^{n}\right)$-symbols have the advantage that the corresponding Hankel operator is continuous:

$$
\begin{aligned}
\left\|T_{f}\right\| & =\sup _{\|g\|_{2}=1}\left\|T_{f}(g)\right\|_{2} \\
& =\sup _{\|g\|_{2}=1}\|P(f g)\|_{2} \leq \sup _{\|g\|_{2}=1}\|(f g)\|_{2} .
\end{aligned}
$$

So we have

$$
\begin{aligned}
\left\|T_{f}\right\| & \leq \sup _{\|g\|_{2}=1}\|(f g)\|_{2} \leq \sup _{\|g\|_{2}=1}\|(g)\|_{2}\|f\|_{\infty} \\
& =1\|f\|_{\infty} .
\end{aligned}
$$


Here $T_{f}$ is the corresponding Toeplitz operator

$$
\begin{aligned}
& T_{f}: \mathcal{F}^{m} \rightarrow \mathcal{F}^{m}, \\
& T_{f} g=P(f g) .
\end{aligned}
$$

It is easy to see that $H_{f}$ is continuous as well. We are interested in the compactness of the operators $H_{f}$. In [24] it is shown that, for $f \in L^{\infty}\left(\mathbb{C}^{n}\right), H_{f}$ is compact if and only if

$$
\left\|f \circ \tau_{\lambda}-P\left(f \circ \tau_{\lambda}\right)\right\|_{2} \rightarrow 0
$$

if $\|\lambda\| \rightarrow \infty$. Here $\tau_{\lambda}(z)=z+\lambda$.

In the next section we will investigate the compactness and continuity of Hankel operators with the symbols $\bar{z}^{n}$ in the spaces $\mathcal{F}^{m}$.

\section{Compactness of $H_{\bar{z}^{m}}$}

Here we will investigate continuity and compactness of Hankel operators with the symbols $\bar{z}^{m}$. One difficulty arising in the investigation is that the multiplication operator $M_{\bar{z}^{m}}$ with $\bar{z}^{m}$ is not globally defined as an operator from $\mathcal{F}^{m}$ to $L^{2}\left(\mathbb{C},|z|^{m}\right)$; there exist $f \in \mathcal{F}$ with $M_{\bar{z}^{m}} f \notin L^{2}\left(\mathbb{C},|z|^{m}\right)$, and

$$
H_{\bar{z}^{m}}=(I-P) M_{\bar{z}^{m}} \text {. }
$$

For more details see [3]. In [23] we have seen that the Hankel operator with the symbol $\bar{z}$ can be interpreted as a globally defined operator even in the case of several variables. There we used facts from Hörmander theory and the identification of the solution operator and the Hankel operator. Here this is not possible.

First we need some definitions.

Definition 1. Let $0<\rho<1$ and $k \in \mathbb{N}$. Then we define

$$
f_{\rho}(z):=f(\rho z)
$$

and

$$
\tilde{f}_{\rho, k}(z)=\bar{z}^{k} f_{\rho}(z) .
$$

Proposition 1. Let $f \in \mathcal{F}^{m}$ and $m \geq 1$. Then

$$
\tilde{f}_{\rho, k}(z)=\bar{z}^{k} f_{\rho}(z) \in L^{2}\left(\mathbb{C},|z|^{m}\right) .
$$

Proof. For $f=\sum_{i=0}^{\infty} a_{i} z^{i}$ we have

$$
\begin{aligned}
\tilde{f}_{\rho, k}(z) & =\bar{z}^{k} \sum_{i=0}^{\infty} a_{i} z^{i} \rho^{i} \\
& =\sum_{i=0}^{\infty} a_{i} \bar{z}^{k} z^{i} \rho^{i} .
\end{aligned}
$$

Furthermore,

$$
\left(\tilde{f}_{\rho}, \tilde{f}_{\rho}\right)=\int_{\mathbb{C}}\left(\sum_{i=0}^{\infty} a_{i} z^{i} \rho^{i} \bar{z}^{k}\right) \overline{\left(\sum_{i=0}^{\infty} a_{i} z^{i} \rho^{i} \bar{z}^{k}\right)} e^{-|z|^{2}} d \lambda(z) .
$$

Using routine calculations as in [3], one can see that

$$
\left(\tilde{f}_{\rho}, \tilde{f}_{\rho}\right)=\sum_{i} \rho^{2 i}(i+k) !\left|a_{i}\right|^{2} .
$$


Using the boundedness of the sequence

$$
\left\{(i+k) \ldots(i+1) \rho^{2 i}, i \in \mathbb{N}\right\}
$$

and the fact that, when $m=2$,

$$
(f, f)=\sum i !\left|a_{i}\right|^{2}
$$

the proposition follows for $m=2$. To prove the general case one has to replace $(i+k)$ ! by $\Gamma\left(\frac{i+k+2}{m}\right)$. Therefore, one has to use Stirling's formula, and the calculations get messy.

Remark. Proposition 1 will be the main ingredient in the following investigation.

Interestingly, the sequence $\frac{c_{k+n, m}^{2}}{c_{k, m}^{2}}-\frac{c_{k, m}^{2}}{c_{k-n, m}^{2}}$ will play a very important role. The following proposition describes its limit behavior.

Proposition 2. Let $m>2 n$. Then $\frac{c_{k+n, m}^{2}}{c_{k, m}^{2}}-\frac{c_{k, m}^{2}}{c_{k-n, m}^{2}} \rightarrow 0$.

Proof. Let us consider the sequence

$$
\frac{c_{k+n}^{2}}{c_{k}^{2}}-\frac{c_{k}^{2}}{c_{k-n}^{2}} .
$$

We infer that this sequence is

$$
\frac{\Gamma\left(\frac{2 k+2 n+2}{m}\right)}{\Gamma\left(\frac{2 k+2}{m}\right)}-\frac{\Gamma\left(\frac{2 k+2}{m}\right)}{\Gamma\left(\frac{2 k-2 n}{m}\right)} .
$$

The limiting behavior of this sequence, due to Stirling's formula $(24]) \Gamma(n+1) \approx$ $\left(\frac{n}{e}\right)^{n} \sqrt{2 \pi n}$, is equivalent to that of

$$
\frac{\left(\frac{2 k+2 n+2}{m}-1\right)^{\frac{2 k+2 n+2}{m}-1} e^{-\frac{2 n}{m}}}{\left(\frac{2 k+2}{m}-1\right)^{\frac{2 k+2}{m}-1}}-\frac{\left(\frac{2 k+2}{m}-1\right)^{\frac{2 k+2}{m}-1}}{\left(\frac{2 k-2 n}{m}-1\right)^{\frac{2 k-2 n}{m}-1} e^{\frac{2 n}{m}}} .
$$

Routine calculation shows that we just have to study the limit behavior of

$$
\left(\frac{2 k+2 n+2}{m}-1\right)^{\frac{2 n}{m}}-\left(\frac{2 k+2 n}{m}-1\right)^{\frac{2 n+2}{m}} .
$$

It is clear that this sequence tends to 0 if $\frac{2 n}{m}<1$.

Theorem 1. Let $n, m \in \mathbb{N}$. Then

$$
\begin{aligned}
& \int_{\mathbb{C}}\left|\tilde{f}_{\rho, n}(z)-P\left(\tilde{f}_{\rho, n}\right)(z)\right|^{2} e^{-|z|^{m}} d \lambda(z) \\
& =\sum_{k=0}^{n-1}\left|a_{k}\right|^{2} c_{k+n}^{2} \rho^{2 k}+\sum_{k=n}^{\infty}\left|a_{k}\right|^{2} c_{k}^{2} \rho^{2 k}\left(\frac{c_{k+n}^{2}}{c_{k}^{2}}-\frac{c_{k}^{2}}{c_{k-n}^{2}}\right),
\end{aligned}
$$

for each $0<\rho<1$ and for each $f(z)=\sum_{k=0}^{\infty} a_{k} z^{k} \in \mathcal{F}^{m}$. Here we abbreviate $c_{k, m}=c_{k}$.

Proof. Since $f$ is holomorphic, it has a Taylor expansion

$$
f(z)=\sum_{k=0}^{\infty} a_{k} z^{k}
$$


Using

$$
P(g)(w)=\int_{\mathbb{C}} g(z) K_{m}(w, z) e^{-|z|^{m}} d \lambda(z),
$$

we have

$$
\begin{aligned}
P\left(\tilde{f}_{\rho, n}\right)(z) & =\int_{\mathbb{C}} \sum_{k=0}^{\infty} \frac{z^{k} \bar{w}^{k}}{c_{k}^{2}}\left(\bar{w}^{n} \sum_{j=0}^{\infty} a_{j} w^{j} \rho^{j}\right) e^{-|w|^{m}} d \lambda(z) \\
& =\int_{\mathbb{C}} \sum_{k, j=0}^{\infty} \frac{z^{k} \bar{w}^{k+n}}{c_{k}^{2}} a_{j} w^{j} \rho^{j} e^{-|w|^{m}} d \lambda(z) \\
& =\sum_{k, j=0}^{\infty} \frac{1}{c_{k}^{2}} a_{j} z^{k} \rho^{j}\left(w^{j}, w^{k+n}\right)_{\mathcal{F} m}
\end{aligned}
$$

Here we abbreviated $c_{k}=c_{k, m}$. Now using

$$
\left(w^{j}, w^{k+n}\right)_{\mathcal{F} m}=c_{j}^{2} \delta_{j, k+n},
$$

we can see that the right side of the equation for $P\left(\tilde{f}_{\rho, n}\right)(z)$ reduces to

$$
\begin{aligned}
& \sum_{k, j=0}^{\infty} \frac{1}{c_{k}^{2}} a_{j} z^{k} \rho^{j}\left(w^{j}, w^{k+n}\right)_{\mathcal{F}^{m}} \\
& =\sum_{j=n}^{\infty} a_{j} \frac{c_{j}^{2}}{c_{j-n}^{2}} \rho^{j} z^{j-n} .
\end{aligned}
$$

From this we have

$$
\begin{aligned}
& \int_{\mathbb{C}}\left|\tilde{f}_{\rho, n}(z)-P\left(\tilde{f}_{\rho, n}\right)(z)\right|^{2} e^{-|z|^{m}} d \lambda(z) \\
& =\int_{\mathbb{C}}\left(\bar{z}^{n} \sum_{k=0}^{\infty} a_{k} z^{k} \rho^{k}-\sum_{k=n}^{\infty} a_{k} \frac{c_{k}^{2}}{c_{k-n}^{2}} \rho^{k} z^{k-n}\right) \\
& \times\left(\bar{z}^{n} \sum_{k=0}^{\infty} a_{k} z^{k} \rho^{k}-\sum_{k=n}^{\infty} a_{k} \frac{c_{k}^{2}}{c_{k-n}^{2}} \rho^{k} z^{k-n}\right) e^{-|z|^{m}} d \lambda(z) \\
& =\int_{\mathbb{C}} \sum_{k=0}^{\infty}\left(\left|a_{k}\right|^{2}|z|^{2 k+2 n} \rho^{2 k}-2 \sum_{k=n}^{\infty}\left|a_{k}\right|^{2} \frac{c_{k}^{2}}{c_{k-n}^{2}} \rho^{2 k}|z|^{2 k}\right. \\
& \left.+\sum_{k=n}^{\infty}\left|a_{k}\right|^{2} \frac{c_{k}^{4}}{c_{k-n}^{4}} \rho^{2 k}|z|^{2 k-2 n}\right) e^{-|z|^{m}} d \lambda(z) \\
& =\sum_{k=0}^{n-1}\left|a_{k}\right|^{2} c_{k+n}^{2} \rho^{2 k}+\sum_{k=n}^{\infty}\left|a_{k}\right|^{2} c_{k}^{2} \rho^{2 k}\left(\frac{c_{k+n}^{2}}{c_{k}^{2}}-\frac{c_{k}^{2}}{c_{k-n}^{2}}\right) .
\end{aligned}
$$

Theorem 2. Let $n \in \mathbb{N}$ and $m>2 n$. Then there is a constant $C_{m}>0$ such that

$$
\int_{\mathbb{C}}\left|\tilde{f}_{\rho, n}(z)-P\left(\tilde{f}_{\rho, n}\right)(z)\right|^{2} e^{-|z|^{m}} d \lambda(z) \leq C_{m} \int_{\mathbb{C}}|f(z)|^{2} e^{-|z|^{m}} d \lambda(z)
$$

for each $0<\rho<1$ and for each $f \in \mathcal{F}^{m}$. 
Proof. Remember (Theorem 1)

$$
\begin{gathered}
\int_{\mathbb{C}}\left|\tilde{f}_{\rho, n}(z)-P\left(\tilde{f}_{\rho, n}\right)(z)\right|^{2} e^{-|z|^{m}} d \lambda(z) \\
=\sum_{k=0}^{n-1}\left|a_{k}\right|^{2} c_{k+n}^{2} \rho^{2 k}+\sum_{k=n}^{\infty}\left|a_{k}\right|^{2} c_{k}^{2} \rho^{2 k}\left(\frac{c_{k+n}^{2}}{c_{k}^{2}}-\frac{c_{k}^{2}}{c_{k-n}^{2}}\right) .
\end{gathered}
$$

The theorem follows from the fact that the sequence

$$
\left(\frac{c_{k+n}^{2}}{c_{k}^{2}}-\frac{c_{k}^{2}}{c_{k-n}^{2}}\right)
$$

is bounded, and from the equality

$$
\int_{\mathbb{C}}|f(z)|^{2} e^{-|z|^{m}} d \lambda(z)=\sum_{k=0}^{\infty}\left|a_{k}\right|^{2} c_{k}^{2} .
$$

Now we want to show that the last theorem implies the continuity of the Hankel operators of the form $H_{\bar{z}^{n}}$ in the topology of $\mathcal{F}^{m}$, where $m>2 n$.

Theorem 3. Let $n \in \mathbb{N}$, and let $\mathcal{F}^{m}$ be defined as above. Furthermore let $m>2 n$. Then the Hankel operator with symbol $\bar{z}^{n}$,

$$
H_{\bar{z}^{n}}: \mathcal{F}^{m} \rightarrow \mathcal{F}^{m \perp}
$$

is bounded on polynomials and therefore can be seen as a globally defined operator. Furthermore, it has the form

$$
f \mapsto F
$$

where

$$
f(z)=\sum_{k=0}^{\infty} a_{k} z^{k}
$$

and

$$
F(z)=\lim _{l \rightarrow \infty}\left(\bar{z}^{n} \sum_{k=0}^{l} a_{k} z^{k}-\sum_{k=n}^{l} a_{k} \frac{c_{k}^{2}}{c_{k-n}^{2}} z^{k-n}\right) .
$$

Proof. The second statement of the theorem is obvious from the proof of Theorem 2.

For the first part we just have to make use of Fatou's theorem:

$$
\begin{aligned}
& \int_{\mathbb{C}} \lim _{\rho \rightarrow 1}\left|\tilde{f}_{\rho, n}(z)-P\left(\tilde{f}_{\rho, n}\right)(z)\right|^{2} e^{-|z|^{m}} d \lambda(z) \\
& \leq \sup _{0<\rho<1} \int_{\mathbb{C}}\left|\tilde{f}_{\rho, n}(z)-P\left(\tilde{f}_{\rho, n}\right)(z)\right|^{2} e^{-|z|^{m}} d \lambda(z) \\
& \leq C_{m} \int_{\mathbb{C}}|f(z)|^{2} e^{-|z|^{m}} d \lambda(z) .
\end{aligned}
$$

Hence

$$
F(z)=\lim _{l \rightarrow \infty}\left(\bar{z}^{n} \sum_{k=0}^{l} a_{k} z^{k}-\sum_{k=n}^{l} a_{k} \frac{c_{k}^{2}}{c_{k-n}^{2}} z^{k-n}\right)
$$


is in $L^{2}\left(\mathbb{C}^{n},|z|^{m}\right)$ and

$$
\int_{\mathbb{C}}|F(z)|^{2} e^{-|z|^{m}} d \lambda(z) \leq C_{m} \int_{\mathbb{C}}|f(z)|^{2} e^{-|z|^{m}} d \lambda(z),
$$

and hence the Hankel operator is bounded.

Up to this moment all results are valid in the case of several dimensions as well. The only difference is that one has to use the multi-index notation, and therefore some notational problems arise. Now we will investigate the compactness of the Hankel operators discussed above. Intuitively speaking, one has to expect that compactness fails in the case of several dimensions. We will restrict our attention to the case of one complex dimension in the following investigation.

Proposition 3. Let $f=z^{j}$ where $j \in \mathbb{N}$. Furthermore, let $n, m \in \mathbb{N}$ and $j \geq n$. Then

$$
P\left(f \overline{z^{n}}\right)(w)=\frac{c_{j, m}^{2}}{c_{j-n, m}^{2}} w^{j-n}
$$

Proof. We have

$$
\begin{aligned}
P\left(f \overline{z^{n}}\right)(w) & =\int_{\mathbb{C}} K(w, z) f(z) \bar{z}^{n} e^{-|z|^{m}} d \lambda(z) \\
& =\int_{\mathbb{C}} \sum \frac{\bar{z}^{i}}{c_{i}^{2}} w^{i} \overline{z^{n}} z^{j} e^{-|z|^{m}} d \lambda(z) \\
& =\int_{\mathbb{C}} \sum \frac{\bar{z}^{i+n} z^{j}}{c_{i}^{2}} w^{i} e^{-|z|^{m}} d \lambda(z) \\
& =\sum \frac{1}{c_{i}^{2}} w^{i} \int_{\mathbb{C}} \bar{z}^{i+n} z^{j} e^{-|z|^{m}} d \lambda(z) \\
& =\sum \frac{1}{c_{i}^{2}} w^{i}\left(z^{j}, z^{i+n}\right)_{\mathcal{F}} \\
& =\sum \frac{c_{i+n}^{2}}{c_{i}^{2}} \delta_{i+n, j} w^{i}=\frac{c_{j}^{2}}{c_{j-n}^{2}} w^{j-n} .
\end{aligned}
$$

Proposition 4. Let $n, m \in \mathbb{N}, i \geq n \in \mathbb{N}$ and $u_{i}(z)=\frac{z^{i}}{c_{i}}$. Then

$$
H_{\bar{Z}^{n}}^{*} H_{\bar{Z}^{n}}\left(u_{i}\right)=\left(\frac{c_{i+n, m}^{2}}{c_{i, m}^{2}}-\frac{c_{i, m}^{2}}{c_{i-n, m}^{2}}\right) u_{i}(w) .
$$

Proof. Direct calculation with $S_{1}\left(u_{i}\right):=H_{\bar{z}^{n}}\left(u_{i}\right)$ shows that

$$
\begin{aligned}
S_{1}^{*} S_{1}\left(u_{i}\right)(w)= & \int_{\mathbb{C}} K_{m}(w, z)\left(\bar{z}^{n} u_{i}(z)-\frac{c_{i} z^{i-n}}{c_{i-n}^{2}}\right)(z-w) e^{-|z|^{m}} d \lambda(z) \\
= & \int_{\mathbb{C}}\left(\sum \frac{\bar{z}^{k}}{c_{k}} \frac{w^{k}}{c_{k}}\right) \\
& \times\left(\bar{z}^{n} u_{i}(z)-\frac{c_{i} z^{i-n}}{c_{i-n}^{2}}\right)(z-w) e^{-|z|^{m}} d \lambda(z)
\end{aligned}
$$


and

$$
\begin{aligned}
\int_{\mathbb{C}}\left(\sum \frac{w^{k}}{c_{k}} \frac{\bar{z}^{k}}{c_{k}}\right)\left(\bar{z}^{n} u_{i}(z)-\frac{c_{i} z^{i-n}}{c_{i-n}^{2}}\right)\left(z^{n}\right) e^{-|z|^{m}} d \lambda(z) \\
=\int_{\mathbb{C}} \frac{z^{i+n}}{c_{i}}\left(\sum \frac{w^{k}}{c_{k}} \frac{\bar{z}^{k+n}}{c_{k}}\right) e^{-|z|^{m}} d \lambda(z) \\
-\frac{c_{i}}{c_{i-n}^{2}} \int_{\mathbb{C}} z^{i}\left(\sum \frac{w^{k}}{c_{k}} \frac{\bar{z}^{k}}{c_{k}}\right) e^{-|z|^{m}} d \lambda(z) \\
=\frac{w^{i}}{c_{i}^{3}} \int_{\mathbb{C}}|z|^{2(i+n)} e^{-|z|^{m}} d \lambda(z)-\frac{w^{i}}{c_{i-n}^{2} c_{i}} \int_{\mathbb{C}}|z|^{2 i} e^{-|z|^{m}} d \lambda(z) \\
=\left(\frac{c_{i+n}^{2}}{c_{i}^{2}}-\frac{c_{i}^{2}}{c_{i-n}^{2}}\right) u_{i}(w) .
\end{aligned}
$$

Remark. It is easily seen that

$$
P\left(z^{i} \overline{z^{n}}\right)(w)=\int_{\mathbb{C}}\left(\sum \frac{w^{k}}{c_{k}} \frac{\bar{z}^{k}}{c_{k}}\right)\left(\bar{z}^{n}\right)\left(z^{i}\right) e^{-|z|^{m}} d \lambda(z)=0
$$

if $i<n$.

Theorem 4. Let $n \in \mathbb{N}$ and $m>2 n$. Then the Hankel operator

$$
H_{\bar{z}^{n}}: \mathcal{F}^{m} \rightarrow \mathcal{F}^{m \perp}
$$

is compact.

Proof. We will make use of the fact that $H_{\bar{z}^{n}}$ is compact $\Leftrightarrow H_{\bar{z}^{n}}^{*} H_{\bar{z}^{n}}$ is. It follows from Theorem 3 that for $i \geq n$,

$$
H_{\bar{z}^{n}}^{*} H_{\bar{z}^{n}}\left(u_{i}\right)=\left(\frac{c_{i+n}^{2}}{c_{i}^{2}}-\frac{c_{i}^{2}}{c_{i-n}^{2}}\right) u_{i} .
$$

Here

$$
\left\{u_{i}(z), i \in \mathbb{N}\right\}=\left\{\frac{z^{i}}{c_{i}}, i \in \mathbb{N}\right\} .
$$

Again using Stirling's formula

$$
\Gamma(n+1) \approx\left(\frac{n}{e}\right)^{n} \sqrt{2 \pi n}
$$

we infer that

$$
\begin{aligned}
& \frac{c_{i+n, m}^{2}}{c_{i, m}^{2}}-\frac{c_{i, m}^{2}}{c_{i-n, m}^{2}} \\
& \approx \frac{\left(\frac{\frac{2 k+4 n}{m}-1}{e}\right)^{\frac{2 k+4 n}{m}-1} \sqrt{2 \pi\left(\frac{2 k+4 n}{m}-1\right)}}{\left(\frac{\frac{2 k+2 n}{m}-1}{e}\right)^{\frac{2 k+2 n}{m}-1} \sqrt{2 \pi\left(\frac{2 k+2 n}{m}-1\right)}}-\frac{\left(\frac{\frac{2 k+2 n}{m}-1}{e}\right)^{\frac{2 k+2 n}{m}-1} \sqrt{2 \pi\left(\frac{2 k+2 n}{m}-1\right)}}{\left(\frac{\frac{2 k}{m}-1}{e}\right)^{\frac{2 k}{m}-1} \sqrt{2 \pi\left(\frac{2 k}{m}-1\right)}} .
\end{aligned}
$$

We have $\frac{c_{i+n, m}^{2}}{c_{i, m}^{2}}-\frac{c_{i, m}^{2}}{c_{i-n, m}^{2}} \rightarrow 0$ as $i \rightarrow \infty$.

So we are finished. 
Lemma 1. The Hankel operator $(m>2 n) H_{\bar{z}^{n}}: \mathcal{F}^{m} \rightarrow \mathcal{F}^{m \perp}$ is Hilbert-Schmidt if and only if $\lim _{i \rightarrow \infty} \frac{c_{i+n}^{2}}{c_{i}^{2}}<\infty$.

Proof. Direct calculation shows that for $k \geq n$ (we abbreviate $c_{n, m}=c_{n}$ )

$$
\begin{aligned}
\left\|H_{\bar{z}^{n}}\left(u_{k}\right)\right\|^{2}= & \frac{1}{c_{k, m}^{2}} \int_{\mathbb{C}}\left|\bar{z}^{n} z^{k}-\frac{c_{k, m}^{2}}{c_{k-n, m}} z^{k-n}\right| e^{-|z|^{m}} d \lambda(z) \\
= & \frac{1}{c_{k, m}^{2}} \int_{\mathbb{C}}|z|^{2 k-2 n}\left(|z|^{4 n}-\frac{2 c_{k}^{2}|z|^{2 n}}{c_{k-n}^{2}}+\frac{c_{k}^{4}}{c_{k-n}^{4}}\right) e^{-|z|^{m}} d \lambda(z) \\
= & \frac{1}{c_{k, m}^{2}} \int_{\mathbb{C}}|z|^{2 k+2 n} e^{-|z|^{m}} d \lambda(z)-\frac{2}{c_{k-n}^{2}} \int_{\mathbb{C}}|z|^{2 k} e^{-|z|^{m}} d \lambda(z) \\
& +\frac{c_{k}^{2}}{c_{k-n}^{4}} \int_{\mathbb{C}}|z|^{2 k-2 n} e^{-|z|^{m}} d \lambda(z) \\
= & \frac{c_{k+n}^{2}}{c_{k}^{2}}-\frac{c_{k}^{2}}{c_{k-n}^{2}} .
\end{aligned}
$$

Since it is known that an operator is Hilbert-Schmidt if and only if

$$
\sum_{k=0}^{\infty}\left\|H_{\bar{z}^{n}}\left(u_{k}\right)\right\|^{2}<\infty
$$

the theorem follows easily.

Theorem 5. The Hankel operator $H_{\bar{z}^{n}}$ is not Hilbert-Schmidt as an operator from $\mathcal{F}^{m}$ to $\mathcal{F}^{m \perp}$.

Proof. The theorem follows from Lemma 1 and the fact that

$$
\frac{c_{i+n, m}^{2}}{c_{i, m}^{2}} \rightarrow \infty .
$$

Corollary 1. Let $\phi_{k}$ be a complete orthonormal system of $\mathcal{F}$. Then

$$
\int_{\mathbb{C}} \int_{\mathbb{C}}\left|z^{n}-w^{n}\right|^{2}\left|\sum_{k} \phi_{k}(z) \overline{\phi_{k}(w)}\right|^{2} e^{-|z|^{m}} e^{-|w|^{m}} d \lambda(z) d \lambda(z)=\infty
$$

and, equivalently,

$$
\int_{\mathbb{C}} \int_{\mathbb{C}}\left|z^{n}-w^{n}\right|^{2}|K(z, w)|^{2} e^{-|z|^{m}} e^{-|w|^{m}} d \lambda(z) d \lambda(z)=\infty .
$$

Proof. We just have to prove the second equality, because $K(z, w)=\sum_{k} \phi_{k}(z) \overline{\phi_{k}(w)}$. Suppose that the kernel belongs to $L^{2}(\mathbb{C} \times \mathbb{C}, \mu \otimes \mu)$. Then the corresponding integral operator is a Hilbert-Schmidt operator, which would imply that the restriction to $\mathcal{F}^{m}$ is also Hilbert-Schmidt. But this restriction coincides with the Hankel operator $H_{\bar{z}^{m}}$, which we already know is not Hilbert-Schmidt.

\section{Corollary 2.}

$$
\int_{\mathbb{C}} \int_{\mathbb{C}}\left|z^{n}-w^{n}\right|^{2}\left|\sum_{k} \frac{z^{k}}{\sqrt{k !}} \frac{\bar{w}^{k}}{\sqrt{k !}}\right|^{2} e^{-|z|^{2}} e^{-|w|^{2}} d \lambda(z) d \lambda(z)=\infty
$$


and, equivalently,

$$
\int_{\mathbb{C}} \int_{\mathbb{C}}\left|z^{n}-w^{n}\right|^{2}\left|e^{z \bar{w}}\right|^{2} e^{-|z|^{2}} e^{-|w|^{2}} d \lambda(z) d \lambda(z)=\infty .
$$

Proof. The first equation follows from Corollary 1. The second equality follows from $\sum_{k} \frac{z^{k}}{\sqrt{k !}} \frac{\bar{w}^{k}}{\sqrt{k !}}=e^{z \bar{w}}$.

\section{ACKNOWLEDGMENTS}

I would like to thank Prof. Haslinger for his guidance during my thesis (supported by FWF (P15279)) and for introducing me to the theory of several complex variables.

\section{REFERENCES}

[1] J. Arazy, S. Fischer and J. Peetre, Hankel operators on weighted Bergman spaces, Amer. J. of Math. 110 (1988), 989-1054. MR 90a:47067

[2] S. Axler, The Bergman space, the Bloch space, and commutators of multiplication operators, Duke Math. J. 53 (1986), 315-332. MR 87m:47064

[3] V. Bargmann, On a Hilbert space of analytic functions and an associated integral transform, Comm. Pure Appl. Math. 14 (1961), 187-214. MR 28:486

[4] F. F. Bonsall, Hankel operators on the Bergman space for the disc, J. London Math. Soc. (2) 33 (1986), 355-364. MR 88e:47045

[5] David Catlin, Global regularity of the $\bar{\partial}-$ Neumann problem, Proceedings of Symposia in Pure Mathematics, vol. 41, Amer. Math. Soc., Providence, RI, 1984, pp. 39-49. MR 85j:32033

[6] David Catlin and John P. D'Angelo, Positivity conditions for bihomogeneous polynomials, Math. Res. Lett. 4 (1997), 555-567. MR 98e:32023

[7] G. B. Folland and J. J. Kohn, The Neumann problem for the Cauchy-Riemann complex, Annals of Mathematics Studies, no. 75, Princeton University Press, Princeton, NJ, 1972. MR 57:1573

[8] S. Fu and E. Straube, Compactness in the $\bar{\partial}-$ Neumann problem on convex domains, J. Functional Analysis 159 (1998), 629-641. MR 99h:32019

[9] S. Fu and E. Straube, Compactness in the $\bar{\partial}$-Neumann problem, Complex Analysis and Geometry (Columbus, OH, 1999), Ohio State Univ. Math. Res. Inst. Publ., 9, de Gruyter, Berlin, pp. 141-160, 2001.

[10] F. Haslinger, Weighted spaces of entire functions, Indiana Univ. Math. J. 35 (1986), 193-208. MR 87f:46043

[11] F. Haslinger, The canonical solution operator to $\bar{\partial}$ restricted to Bergman spaces, Proc. Amer. Math. Soc. 129 (2001), 3321-3329. MR 2003c:32036

[12] F. Haslinger, The canonical solution operator to $\bar{\partial}$ restricted to spaces of entire functions, Ann. Fac. Sci. Toulouse Math. (6) 11 (2002), 57-70.

[13] H. Heuser, Funktionalanalysis, B. G. Teubner, Stuttgart, 1992. MR 94d:46001

[14] L. Hörmander, $L^{2}$ estimates and existence theorems for the $\bar{\partial}$ operator, Acta Mathematica 113 (1965), 89-152. MR 31:3691

[15] L. Hörmander, An introduction to complex analysis in several variables, D. Van Nostrand, Princeton, NJ, 1966. MR 34:2933

[16] S. Janson, Hankel operators between weighted Bergman spaces, Ark. Mat. 26 (1988), 205-219. MR 91j: 47027

[17] J. J. Kohn and L. Nirenberg, Non-coercive boundary value problems, Comm. Pure Appl. Math. 18 (1965), 443-492. MR 31:6041

[18] J. J. Kohn, Harmonic integrals on strongly pseudo-convex manifolds. I, Ann. of Math. (2) 78 (1963), 112-148. MR 27:2999

[19] Steven G. Krantz, Compactness of the $\bar{\partial}-$ Neumann operator, Proc. Amer. Math. Soc. 103 (1988), 1136-1138. MR 89f:32032

[20] R. Meise and D. Vogt, Einführung in die Funktionalanalysis, Vieweg, Braunschweig, 1992. MR 94f: 46003 
[21] R. Rochberg, Trace ideal criteria for Hankel operators and commutators, Indiana Univ. Math. J. 31 (1982), 913-925. MR 84d:47036

[22] W. Rudin, Functional Analysis, McGraw-Hill, New York, 1973. MR 51:1315

[23] G. Schneider, Compactness of the solution operator to $\bar{\partial}$ on the Fock space in several dimensions, ESI-preprint 1206, 2002.

[24] K. Stroethoff, Hankel and Toeplitz operators on the Fock space, Michigan Math. J. 39 (1992), 3-16. MR 93d:47058

[25] K. Stroethoff, Compact Hankel operators on the Bergman space, Illinois J. Math. 34 (1990), 159-174. MR 91a:47030

[26] K. Stroethoff, Compact Hankel operators on the Bergman spaces of the unit ball and polydisc in $\mathbb{C}^{n}$, J. Operator Theory 23 (1990), 153-170. MR 91i:47040

[27] R. Wallsten, Hankel operators between weighted Bergman spaces in the ball, Ark. Mat. 28 (1990), 183-192. MR 91i:47041

[28] J. Weidmann, Lineare Operatoren in Hilberträumen, B. G. Teubner, Stuttgart, Leipzig, Wiesbaden, 2000. MR 2002m:47001]

[29] Ke He Zhu, Hilbert-Schmidt Hankel operators on the Bergman space, Proc. Amer. Math. Soc. 109 (1990), 721-730. MR 90k:47060

Institut für Mathematik, Universität Wien, Strudlhofgasse 4, A-1090 Wien, Austria E-mail address: georg.schneider@univie.ac.at

Current address: Institut für Betriebswirtschaftslehre, Universität Wien, Brünner Strasse 72, A-1210 Wien, Austria 